\title{
Aportaciones y Límites del Uso de la Abordaje Centrada en la Persona (ACP) para la Fundamentación Teórica de la Consejería en VIH / SIDA
}

\author{
Ligia Rivero Pupo ${ }^{1}$ \\ Instituto de Saúde da Secretaria de Estado de Saúde de São Paulo, São Paulo, Brasil \\ José Ricardo Carvalho Mesquita Ayres \\ Faculdade de Medicina da Universidade de São Paulo, São Paulo, Brasil
}

\section{Resumen}

Estudios nacionales e internacionales indican que a consejería en ETS/sida presenta fragilidades y problemas tales como la carencia de fundamentación teórica o inconsistencias entre bases teóricas y desdoblamientos técnicos. Este estudio examinó contribuciones de uno de los marcos teóricos que influyeron en la construcción de estrategias de consejería en ETS en Brasil, el Abordaje Centrado en la Persona (ACP), de Carl Rogers. Se analizaron las limitaciones de su uso en el ámbito de las ETS/sida, a partir del análisis de documentos producidos por la Organización Mundial de la Salud, el Programa de Sida de Naciones Unidas y el Ministerio de Salud de Brasil. El material documental fue tratado desde el punto de vista interpretativo, cotejando las construcciones conceptuales rogerianas con los componentes del proceso de trabajo recomendado en los documentos, examinándose la consistencia y coherencia internas. Fueron evidenciadas incompatibilidades en relación a: objetivos, foco, resultados esperados y postura del profesional. Tales desacuerdos parecen ser consecuencia de diferencias entre las finalidades originales de la consejería basada en la ACP y aquellas requeridas en las estrategias de prevención de ETS/sida. La confluencia de diversas finalidades confiadas a la consejería en el campo de las ETS/sida configura obstáculos para un uso consciente de la ACP. Se señala la pertinencia del abordaje rogeriano en la consejería como técnica de apoyo en la toma de decisiones y el manejo de situaciones cotidianas, pero se apunta a la necesidad de rever objetivos y procedimientos estandarizados y orientados al cambio de comportamiento y a la vigilancia epidemiológica.

Palabras clave: Síndrome de inmunodeficiencia adquirida, enfermedades de transmisión sexual, modelos teóricos, Abordaje Centrada en la Persona, Carl Rogers, estrategias de prevención.

\section{Contribuições e Limites do Uso da Abordagem Centrada na Pessoa para a Fundamentação Teórica do Aconselhamento em DST/Aids}

\section{Resumo}

Mesmo sendo uma importante tecnologia de prevenção, estudos nacionais e internacionais indicam que o aconselhamento no campo das DST/aids, apresenta fragilidades e problemas, entre eles, a carência de fundamentação teórica ou inconsistências entre bases teóricas e desdobramentos técnicos. Este estudo pretendeu examinar as contribuições conceituais de um dos quadros teóricos que influenciaram a construção de estratégias e técnicas de aconselhamento em DST no Brasil, a Abordagem Centrada na Pessoa (ACP), de Carl Rogers, assim como as limitações de seu uso no âmbito das DST/aids, a partir da

Endereço para correspondência: Av. Dr. Arnaldo, 355, Consolação, São Paulo, SP, Brasil 01246-000. E-mail: ligia@isaude.sp.gov.bre jrcayres@usp.br 
análise de documentos produzidos pela Organização Mundial de Saúde, Programa de Aids das Nações Unidas (UNAIDS), e Ministério da Saúde - Brasil, tidos como as principais referências normativas para o aconselhamento. Tratou-se o material documental de forma interpretativa, cotejando-se as construções conceituais rogerianas com os componentes do processo de trabalho recomendado nos documentos, examinando-se sua consistência e coerência internas. Evidenciaram-se incompatibilidades em relação aos objetivos, foco, resultados esperados e postura do profissional. Tais desacordos parecem decorrer de diferenças entre as finalidades originais do aconselhamento baseado na ACP e aquelas demandadas nas estratégias de prevenção às DST/aids. A confluência de diversas finalidades colocadas ao aconselhamento no campo das DST/aids, configuram obstáculos a um uso consistente da ACP. Conclui-se pela pertinência e interesse da abordagem rogeriana no aconselhamento como técnica de apoio para tomada de decisões e manejo de situações cotidianas no campo da prevenção, mas aponta-se a necessidade de rever objetivos e procedimentos padronizados voltados à mudança de comportamento e a vigilância epidemiológica.

Palavras-chave: Aconselhamento, síndrome da imunodeficiência adquirida, doenças sexualmente transmissíveis, modelos teóricos, abordagem centrada na pessoa, Carl Rogers, estratégias de prevenção.

\title{
Contributions and Limits of the Use of Person-Centered Therapy (PCT) for the Theoretical Foundation of STD/Aids Counseling
}

\begin{abstract}
Although it figures as an important prevention technology, national and international studies point out that counseling in the STD/AIDS field present frailties and problems, among them the lack of theoretical grounds or inconsistencies among theoretical bases and technical developments. This study aimed to examine the conceptual contribution of one of the theoretical frames that influenced the construction of counseling strategies and techniques in STD in Brazil, the Person-centered therapy (PCT), developed by Carl Rogers, as well as the limits of its employment in the STD/AIDS context, from the analysis of documents produced by the World Health Organization, the Joint United Nations Program on HIV/ AIDS (UNAIDS), and Ministry of Health - Brazil, regarded as the main normative references for counseling. The documentary material was approached interpretively, the rogerian conceptual constructions compared with the work process recommended in the documents, examining its consistence and internal coherence. Incompatibilities were evidenced regarding their objectives, focus, expected results and professional attitude. Such disagreements seem to stem from differences among the original aims of PCT-based counseling and those demanded in STD/AIDS prevention strategies. The confluence of many goals added to counseling in the field of STD/AIDS presented complications to a consistent use of the PCT. The study agrees with the pertinence and interest of the rogerian approach in counseling as a support technique for the decision making and management of everyday situations in the field of prevention, but the need to revise objectives and standardized procedures focused on behavioral change and epidemiological vigilance is also stressed.
\end{abstract}

Keywords: Counseling, acquired immunodeficiency syndrome, sexually transmitted diseases, theoretical models, person-centered therapy, Carl Rogers, prevention strategies.

El término "consejería" está siendo utilizado históricamente para una amplia variedad de actividades e intervenciones que se relacionan principalmente al manejo individualizado de situaciones, desafíos y dilemas de la vida diaria, desde fomentar el desarrollo humano y el bien estar personal. Desde la década de 1920 hasta la década de 1950, se llamó de consejería, la práctica de ayuda enfocada y objetiva, de carácter educacional y preventivo que, por medio del 
descubrimiento, evaluación, realce e incremento de los recursos internos e inter-personales de individuos y grupos, trata de aportar a una mejor calidad de vida, así como mayor satisfacción personal (Whiteley, 1999).

Con el transcurso de los años, este tipo de relación de ayuda empezó a especializarse cada vez más, acumulando distintas técnicas y metodologías, principios y abordajes, basados en diferentes concepciones del ser humano, sobre lo que constituye una relación de ayuda y sus varias comprensiones sobre los procesos de desarrollo de la personalidad, de cambios de conducta y del proceso de enseñanza-aprendizaje.

Pese a que exista cierta variación en la forma en que se ha definido, la consejería ha sido entendida entre distintos autores como una tecnología de ayuda, de cuidado, y como una práctica instrumental que ofrece auxilio estructurado $y$ personalizado para el manejo de situaciones difíciles y de crisis que exigen ajustes y adaptaciones, para resolver problemas específicos y para la toma de decisiones (Corey, 1997; Pattersson \& Eisenberg, 2003; Scheeffer, 1976).

Para ello, la consejería abarca, de forma resumida, las siguientes etapas y tareas: (a) identificación y análisis de problemas y circunstancias específicas de la vida; (b) aumento del discernimiento, conocimiento y conciencia de los diferentes elementos involucrados en dichas situaciones (c) evaluación de las condiciones, recursos (tanto personales como sociales), estrategias, alianzas y obstáculos existentes para su manejo; (d) definición del potencial de cambiar dichas condiciones y actitudes personales; (e) elección y experimentación de acciones específicas, consideradas viables y convenientes para poder transformar la realidad en cuestión (Corey, 1997; Pattersson, 1973; Pattersson \& Eisenberg, 2003; Santos, 1982; Tyler, 1969).

Por ello aporta para que el individuo tenga más elementos para posicionarse ante su situación de vida, elaborando así un plan personalizado de acción, contextualizado y satisfactorio. Para ese fin, esta tecnología se respalda en establecer condiciones favorables para que el individuo evalúe sus problemas y tome decisiones. La concentración es en el manejo de los aspectos sociocul- turales y afectivo-emocionales de las situaciones (World Health Organization [WHO], 1995b).

No obstante, la práctica de la consejería ha presentado imprecisiones y ambigüedades, dificultando y mucho una demarcación clara y la discriminación de sus fronteras y de su identidad específica. Esto se debe parcialmente al hecho de que, desde su origen, se creó en base a cierto grado de hibridismo, aunando saberes tanto del campo de la educación como el de la psicología, y enfocando no solo aspectos cognitivos sino que afectivos y emocionales (Lewis, 1970; Santos, 1982; Scheeffer, 1976; Whiteley, 1999).

La consejería también se ha utilizado como una estrategia para lidiar con la dimensión psicosocial de la epidemia de VIH/Sida. Al principio, en la década de 1980, se utilizó en la situación de pruebas anti-HIV, como un abordaje para hacer frente a la discriminación asociada al Sida, como espacio para poder aclarar y disminuir las barreras que rodeaban la prueba e incrementar la información para la población sobre esta enfermedad y sus formas de prevención. Posteriormente, en el decenio de 1990, fue utilizado para manejar los diferentes límites de esta misma terapéutica (diagnóstico tardío, ausencia de vacunas, efectos secundarios, dificultades en la adherencia al tratamiento).

A lo largo de los años, como parte de la respuesta social que se ofreció mundialmente para hacer frente a esta epidemia, la consejería empezó a asociarse a la "puerta de entrada" a las distintas estrategias de respuesta a la epidemia, al acceso a las distintas estrategias de respuesta a la epidemia: oferta voluntaria e informada de la prueba anti-HIV, tratamiento temprano de esta enfermedad, interrupción y disminución de la cadena de infección, control de la transmisión materno infantil, suministro de orientación calificada, personalizada y contextualizada, y la disminución del estigma, de los prejuicios y demás impactos sociales y personales de esta epidemia (Joint United Nations Programme on HIV/AIDS [UNAIDS], 2000a; WHO, 1995b).

El mismo proceso tuvo lugar en el Brasil, con la consejería que se introdujo a fines de la década de 1980 (Ministerio de la Salud [MS], 1988, 1989, 1993). 
Estudios nacionales e internacionales muestran sin embargo que los profesionales que llevan a cabo la consejería poseen distintos entendimientos y concepciones sobre su estructura y sus objetivos (Centers for Disease Control and Prevention [CDC], 2001) y que existe una considerable variación en la forma como se pone en práctica (Castrucci, Kamb, \& Hunt, 2002; CDC, 2001; Grinstead, 1997; Holtgrave, Reiser, \& Franceisco, 1997; UNAIDS \& WHO, 2000). Se señala que en muchas ocasiones, se tiende a banalizar la consejería y se la considera tan solo como una simple transmisión de informaciones, o como una actividad con formato ya pronto, de orientación estandarizada en función del perfil de riesgo, ignorando su complejidad y especificidad, subestimando su dimensión psicosocial y de cuidado, inherentes a esta actividad (Filgueiras \& Deslandes, 1999). Además de estos problemas, la consejería muy a menudo se confunde con otras prácticas de cuidado, tales como acciones educacionales y de orientación individual sobre procedimientos (Dolcini, Canin, Gandelman, \& Skolnik, 2004).

Aún los manuales y documentos de referencia sufren de una ausencia de fundamentación teórica y técnica clara y precisa, presentando dicha actividad como si fuese una "receta lista", es decir, como un arsenal de contenidos, orientaciones generales e informaciones técnicas consideradas relevantes y que deben reproducirse en las atenciones. Existen, además, pocas evaluaciones sobre la calidad y la efectividad del uso de dicha tecnología (Sikkema \& Bissett, 1997).

La falta de fundamentación teórica y discusión conceptual más consistente hace con que la ejecución de esta actividad sea bastante dependiente de la formación anterior de cada consejero, de su interés y curiosidad intelectual, y de cómo logra sistematizar su propia práctica. La práctica cotidiana de consejería exige algún grado de fundamentación teórica, bajo pena de perder la eficacia tecnológica, de realizarse de forma mecánica o intuitiva. Adicionalmente, las reflexiones teóricas que son la base de esta acción pueden proveer un fundamento para juzgar si todos los elementos necesarios para la intervención se han tenido en cuenta, identificando factores y condiciones que favorecen y que limi- tan su éxito y aplicabilidad, lo cual aporta a una evaluación exacta de cuando y como tal tecnología debe ser utilizada.

La noción en sí de "best practices", o "buenas prácticas", desarrollada por la UNAIDS para evaluar los cuidados médicos, pero aplicada ya a la actividad de consejería, propone que las buenas prácticas son: aquellos procesos o actividades que incorporan los valores, los principios y los conceptos de las teorías de base; que son consistentes con la evidencia científica y que pueden desarrollarse de distintas formas dependiendo del contexto. De esta forma, para seguir rumbo a las prácticas más adecuadas y consistentes, es necesario aclarar muy bien los conceptos, los principios y las opciones metodológicas que le son subyacentes (Myers, Worthington, Haubrich, Ryder, \& Calzavara, 2003).

Si mundialmente la práctica de consejería se considera como siendo tan pertinente en las estrategias de respuesta a la epidemia de VIH/ Sida, entonces nos incumbe cuestionarnos sobre lo que ocurriendo con la consejería en el contexto brasileño. Y si aceptamos que la claridad de sus fundamentos teóricos y su coherencia con las proposiciones técnicas son presupuestos para la calidad de la aplicación de consejería, un punto de partida relevante será argumentar hasta qué punto esta práctica está basada en y es coherente con las orientaciones técnicas que orientan la respuesta brasileña. En este sentido, este artículo tiene la intención de analizar la principal referencia teórica utilizada en las propuestas técnicas de consejería en el campo de las EST/Sida en el Brasil - el Abordaje Centrado en la Persona, de Carl Rogers - identificando tanto los subsidios ofrecidos por esta vertiente teórica para estructurar las prácticas propuestas, así como sus inconsistencias, incoherencias y dificultades en la adopción de dicho referencial teórico en el contexto del Brasil.

\section{Metodo}

Para proseguir con este estudio, se recorrieron las siguientes etapas:

1. Relevamiento y análisis de manuales, documentos normativos y recomendaciones sobre la consejería en EST/Sida nacionales 
e internacionales. El cuerpo documental nacional consiste en todos los manuales y documentos de referencia oficiales producidos entre 1988 y 2011 por la Coordinación Nacional de EST/Sida y que se refieren a la práctica de consejería en EST/Sida. En lo que respecta los internacionales, se seleccionaron los manuales internacionales mencionados como referencia para poder elaborar los manuales nacionales, los que fueron mencionados en la gran mayoría de artículos sobre la consejería en EST/Sida (nacionales e internacionales), y que fueron producidos por organismos internacionales del área - UNAIDS y CDC.

Los manuales y documentos nacionales de referencia relevados fueron: Manual de Condutas Clínicas (MS, 1988); Manual de Consejeria/ SIDA (MS, 1989); Normas de Organización e Funcionamento dos Centros de Orientación e Apoio Sorológico (MS, 1993); Consejeria em EST, HIV e Sida - Diretrizes e Procedimentos Básicos (MS, 1997); Manual de Treinamento de Consejería em EST, HIV e Sida (MS, 1998); Diretrizes dos Centros de Testagem e Consejería - CTA (MS, 2000); Consejería em EST/HIV/Sida para a Atención Básica (MS, 2004); Oficina de Consejería em EST/HIV/ Sida para a Atención Básica (MS, 2005); Relatório do I Encontro de Avaliación de Centros de Apoio Sorológico (MS, 1994); Relatório do II Encontro de Avaliación de Centros de Apoio Sorológico (MS, 1996a); Relatório do Seminário de Consejería (MS, 1996b); Consejeria: Um Desafio para a Prática Integral em Saúde - Avaliación das Ações (MS, 1999).

Los manuales y documentos internacionales de referencia analizados fueron: Guidelines for Counseling about HIV Infection and Disease (WHO, 1990); Technical Guidance on HIV Counseling, Testing e Referral: Standards and Guidelines (CDC, 1993); Technical Guidance on HIV Counseling, Testing e Referral: Standards and Guidelines (CDC, 1994); Revised Guidelines for HIV Counseling, Testing and Referral and Revised Recommendations for HIV Scre- ening of Pregnant Women (CDC, 2001); Counseling for HIV/Aids: A Key to Caring (WHO, 1995a); Source Book for HIV/Aids Counseling Training (WHO, 1995b); Voluntary Counseling and Testing (VCT) Technical Update (UNAIDS, 2000b); Opening up the HIV/Aids Epidemic: Guidance on Encouraging Beneficial Disclosure, Ethical Partner Counseling and Appropriate Use of HIV Case - Reporting (UNAIDS \& WHO, 2000); Tools for Evaluating HIV Voluntary Counseling and Testing (UNAIDS, 2000a); The Impact of Voluntary Counseling and Testing: A Global Review of the Benefits and Challenges (UNAIDS \& WHO, 2001); Increasing Access to HIV Testing and Counseling (WHO, 2002);

2. Identificación de los textos y trechos que se referían al Abordaje Centrado en la Persona de Carl Rogers para servir de base a las recomendaciones propuestas;

3. Revisión del marco conceptual del Abordaje Centrado en la Persona, basado en el estudio de ocho de los doce libros de Rogers, abarcando desde el inicio de su producción hasta sus últimas escrituras. Se estudiaron además doce artículos de distintos períodos de su vida, que discuten: la práctica de la psicoterapia y la relación de ayuda; el proceso de comunicación interpersonal; el proceso de convertirse en persona; la práctica de la ciencia y la práctica de atención clínica; su visión de la educación y de la enseñanza-aprendizaje; y el abordaje de problemas, dificultades y tensiones socioculturales, políticas y comunitarias. Se relevaron y analizaron además doce artículos y un libro escritos por comentadores y críticos del pensamiento de Rogers. La selección y el análisis del material trató de priorizar textos que contribuyesen a aclarar los diversos elementos implicados en el establecimiento de una relación interpersonal de ayuda efectiva, tal como pretende ser la consejería en EST/Sida, y que por lo tanto, discutían y examinaban las características, los componentes, las condiciones y los resultados involucrados en la práctica de una relación de ayuda; 
4. Cotejamiento y comparación de los aportes ofrecidos por esta corriente teórica con las principales recomendaciones suministradas por los manuales y documentos de referencia para instruir a los profesionales sobre la ejecución de esta práctica.

Se trabajó en todo el corpus documental desde una perspectiva comprensivo-interpretativa (Gadamer, 2004). Con la intención de realizar un abordaje hermenéutico de los documentos relevados, el plan de análisis y de investigación de este material salió en la búsqueda de respuestas y preguntas consideradas importantes para la práctica de consejería en EST/ Sida: características de la relación de ayuda, condiciones para su práctica y éxito, papel del profesional, resultados esperados, su formato y sus límites. Nos incumbimos además de tratar de entender, aunque fuese de forma limitada, la tradición de pensamiento y el contexto del cual se origina el pensamiento de Rogers y los textos técnicos trabajados.

De esta forma, se delinearon las siguientes categorías de análisis: (a) concepción de consejería; (b) objetivos y resultados esperados; (c) papel y actitudes del profesional; (d) papel de la persona que recibe la ayuda. Para cada una de estas categorías, se intentó inicialmente apuntar la visión presentada por la teoría de Carl Rogers, enfatizando algunos aportes y límites $\mathrm{y}$, a seguir, se cotejó dicha perspectiva con lo propuesto en manuales de EST/Sida, identificando posibilidades y dificultades en la aplicación de este referencial teórico dentro de este contexto específico.

\section{Contribuciones y Subsidios del Abordaje Centrado en la Persona para la Práctica de Consejería en EST/Sida}

\section{Concepción de Carl Rogers de Consejería y su Aprovechamiento en el Campo de las EST/Sida}

Un primer aspecto que se debe subrayar en el marco de Carl Rogers es la centralidad en la escena de la atención, de la relación humana y de las personas implicadas en esta. Así, pese a que la consejería puede ser considerado como siendo una tecnología, siempre será, ante todo, una relación y un encuentro interpersonal e intersubjetivo (Rogers, 2005b). Esta visión hace hincapié en las subjetividades presentes, es decir, en la dimensión existencial, experimental y simbólica de esa vivencia, de ese encuentro humano, con sus sentidos, sensaciones y significados propios, mostrando cuan decisivos son esos factores para la dirección, intensidad, calidad, relevancia y efectos de esa relación para los individuos involucrados o abarcados por el mismo (Rogers, 2001a). Bajo esta perspectiva, este encuentro no puede reducirse ni a una simple conversación ni a la puesta en práctica rigurosa de una técnica. Es una "forma de ser" y de posicionarse frente al otro, es una vivencia en abierto, indeterminada, que se debe construir, aunque conlleve una intencionalidad y una expectativa previa de ambas partes, y que pueda ser respaldada por saberes técnicos y científicos (Kirschenbaum \& Henderson, 1989).

De esta forma, la consejería no es una actividad con procedimientos determinados o un "modo de hacer" genérico, repetitivo y prescriptivo. No es una técnica o una estrategia previamente dirigida y cerrada en lo que respecta sus horizontes de actuación, y tampoco es un espacio para proveer orientaciones, sugerencias y consejos estructurados y establecidos con antelación. Los contenidos y procedimientos no existen en sí mismos, no son una guía para la acción, sino que necesitan "dejarse" adaptar, y ser modificados y contextualizados a partir de las situaciones, experiencias y proyectos de vida del que recibe la consejería.

Cualquier problema o asunto concreto no debe ser abordado en sí mismo, de manera fragmentada, sino más bien en su relación con la totalidad de la experiencia vivida por la persona - incluyendo los aspectos cognitivos, afectivos, sensoriales, sociales, así como el presente, el pasado y el futuro. Se trata de la persona como un todo, históricamente ubicada, que vive determinados problemas y situaciones y que les otorga determinados significados y valoraciones, que debe ser accedida y alcanzada al abordar esos mismos temas. Hay un ensanchamiento del horizonte normativo que rige esta práctica: el guía 
para las acciónes es la totalidad existencial de las personas involucradas.

Es una relación de ayuda, de provecho y de utilidad para el otro. Pero dicha ayuda en el sentido de propósito y de interés, especialmente para la persona que se pretende ayudar, es decir, se enfoca en la persona del otro, en su alteridad, $\mathrm{y}$ no en los intereses y demandas personales y sociales del profesional de ayuda o del contexto social en donde esta situación se lleva a cabo. Es una práctica que pretende ayudar a otro individuo a ayudarse (Rogers, 2001a).

Adicionalmente, no es un procedimiento que se debe efectuar PARA el otro, sino más bien proveer las condiciones para que el individuo evalúe sus problemas y tome decisiones. Es una tecnología de cuidado, que se respalda en establecer las condiciones favorables dentro de la propia relación interpersonal y comunicativa, $\mathrm{y}$ en el manejo de los aspectos socioculturales $\mathrm{y}$ afectivo-emocionales de las situaciones. Es un tipo de relación que, por sus mismas características, puede ser usada por el otro en su propio beneficio.

En el campo de las EST/Sida, es interesante observar que la concepción de consejería pasó por algunas modificaciones en lo que se refiere a la forma en que fue definida, principalmente por los manuales nacionales, desde 1988 hasta su última versión dirigida a la atención básica en 2005. En los manuales de 1988 y 1989, la consejería era concebida como una estrategia y un procedimiento directivo, enfocado en una aclaración intelectual, en el suministro de informaciones y en proveer sugerencias de comportamiento y de acción, establecidas previamente por las políticas públicas, por los estudios epidemiológicos y los conocimientos existentes sobre prevención, así como por discursos técnico-científicos de las diversas áreas teóricas implicadas (MS, 1988). El manual de 1989 cita, incluso, que el contenido abordado debería ser estandarizado y que se deberían utilizar técnicas uniformes para llevar a cabo esta práctica, pese a que no detalla cuáles serían esas técnicas (MS, 1989).

En una segunda etapa, en el manual de 1993 (Normas de Organización e Funcionamento dos Centros de Orientación e Apoio Sorológico), la consejería no se considera apenas como un es- pacio para proveer informaciones, sino se considera igualmente como una práctica que debe demostrar ser efectiva al motivar para cambios de comportamiento. No obstante, no existe aquí cualquier distinción entre consejería y educación en salud, y este manual parece asociar el cambio de motivación y la capacidad de alterar comportamientos estrictamente al convencimiento intelectual y a la apropiación cognitiva sobre los riesgos de infección por el VIH y, para ello, al acceso a las informaciones técnico-científicas precisas. No da una dirección y ni instruye sobre cómo debería ser un abordaje más personalizado, o como podría realizarse el manejo de aspectos afectivo-emocionales (MS, 1993).

Consejería es un proceso que puede ayudar al individuo a entender mejor y lidiar con sus problemas, convivir con su ambiente social y, cuando necesario, motivarse para un cambio de comportamiento. Se lleva a cabo por medio de la información y educación directa y personal y desempeña un rol crítico en la prevención y control del VIH/SIDA. (MS, 1988, p. 13)

En una tercera etapa, la concepción de consejería en el campo del Sida se amplía y se remodela hacia una noción de cuidado. Esta nueva concepción se presenta a partir del "Consejería em EST, HIV e Sida-Diretrizes e Procedimentos Básicos" de 1997, del "Diretrizes dos Centros de Testagem e Consejeria - CTA" (MS, 2000), y después reforzada por el "Consejeria em EST/ HIV/Sida para a Atención Básica” (MS, 2004). En dicho material, demuestra estar claramente basado e influenciado por el Abordaje Centrado en la Persona, de Carl Rogers.

En estos últimos manuales, la consejería no se considera como siendo un procedimiento estructurado y cerrado, no como una técnica, sino que como un diálogo; una relación comunicativa basada en valores y actitudes que tienen como miras proporcionar condiciones favorables y un entorno propicio para que el mismo individuo evalúe sus riesgos, dificultades y contexto, tome decisiones y encuentre formas satisfactorias de hacer frente a sus problemas. Se define como un proceso individualizado y centrado en el cliente, y busca rescatar su integralidad como persona (MS, 1997, 2004). 
Según esta nueva concepción, la consejería se ha definido como una acción continuada (proceso), centrado no en un problema, o en un contenido específico que se debe transmitir, o aún en una lista de informaciones que se deben recopilar; sino más bien en la persona que busca ayuda - su demanda, motivos por haber venido al centro de atención, percepciones, miedos, dudas, cuestiones, intereses y dificultades concretas. No se considera como un procedimiento que se realiza para el otro, o como la inducción de una acción específica que el otro debe llevar a cabo, sino apenas con una relación que logre propiciar condiciones para que el individuo evalúe sus problemas, situación y riesgos personales, tomando decisiones al respecto de las mismas. Dentro de esa óptica, no es el profesional que evalúa los riesgos para el otro, o que explora los problemas por medio de un cuestionario específico, o brinda las soluciones más adecuadas. El profesional meramente posibilita condiciones para que el individuo pueda hacer eso.

Entendemos como consejería un proceso de escucha activa, individualizado y centrado en el cliente. Presupone la capacidad de establecer una relación de confianza entre los interlocutores, con miras a rescatar los recursos internos del cliente, para que éste mismo tenga la posibilidad de reconocerse como el sujeto de su misma salud y transformación. (MS, 1997, p. 11)

Es un diálogo, basado en una relación de confianza, que objetiva proporcionarle a la persona la posibilidad de evaluar sus propios riesgos, tomar decisiones propias y encontrar formas de hacer frente a sus problemas relacionados a las EST/HIV/Sida (MS, 2004, p. 7).

En los manuales internacionales estudiados se percibe más coherencia y regularidad en la forma en que se concibe la consejería. Desde el inicio de la década de 90, estos manuales contienen una clara y marcada influencia del abordaje de Carl Rogers y, por ello, más apertura para la construcción de una relación no programada, enfocada en la dimensión afectivo-emocional y social de las vivencias de los clientes, y en los significados que cada individuo ha dado a tales vivencias. Tanto el CDC como la UNAIDS proponen desde ese entonces un modelo interactivo de consejería, centrado en la persona que solicita ayuda, distinguiéndolo de una atención individualizada que ofrece mensajes informativos estandarizados de evaluación y reducción de riesgo (CDC, 2001; WHO, 1995b).

Estos manuales distinguen además la acción de consejería de la acción educacional, indicando que, en la consejería, la comunicación es personalizada, confidencial; tiene como objetivo provocar cambios relevantes en la vida personal y ayudar en la solución de problemas individuales; en éste la dimensión informativa esta siempre subordinada y relacionada al manejo de aspectos socioculturales, afectivos y emocionales del diagnóstico, prevención y tratamiento. Por lo tanto, la información sirve a un propósito individual y debe articularse con la experiencia de vida y las necesidades personales de cada individuo (CDC, 2001; WHO, 1995a, 1995b).

Igualmente, hay una explicación más amplia y una discusión sobre las posturas y actitudes que el profesional debe desarrollar en diferentes contextos.

Aunque hubo un cambio significativo en la perspectiva de la concepción y conceptuación de consejería en los manuales brasileños a lo largo del tiempo, se percibe que los mismos manuales no estructuran sus objetivos, los procedimientos y acciones propuestas en base a la definición que plantean.

\section{Objetivos y Resultados Esperados para la Consejería}

El Abordaje Centrado en la Persona (ACP) propone objetivos para la relación de ayuda efectivamente articulados con la persona del otro. La consejería se aleja de los objetivos cerrados y pre-concebidos, para concentrarse en la creación de condiciones favorables para que el mismo individuo analice y entienda su propia realidad, establezca sus metas con respecto a su momento de vida y movilice los recursos para alcanzarlas (Rogers, 2001b, 2005a). De esta manera, los objetivos deben ser siempre estructurados, organizados junto con el otro, es decir, deben ser construidos en la relación. El individuo que pide 
ayuda debe siempre participar en la delimitación de lo que será abordado y de la meta que se debe alcanzar.

Los objetivos de consejería así propuestos se basan en la convicción de que cada persona tiene el derecho de ser corresponsable de la construcción de su atención, y de elegir sus propios fines en la vida, aunque estos sean divergentes de los que hubiera elegido el profesional para él. Además, se admite la imposibilidad, la inutilidad, la ineficiencia, la arbitrariedad y la prepotencia de tratar de generar y conducir diferentes aspectos de la vida de otros, aunque sea con el consentimiento de estos mismos. De esta forma, hace con que la atención sea más flexible al valorar y utilizar las exigencias y la experiencia vivida por cada persona ante una determinada situación, como guía para la construcción de cada sesión.

En relación a los resultados esperados con la tecnología de consejería, la concepción de Rogers de relación de ayuda, sugiere resultados que sean más realistas, especificos, personalizados, acordados y vinculados a la experiencia individual, o sea, una solución que sea adecuada para determinado individuo, en un determinado momento histórico, en determinado contexto $-\mathrm{y}$ no genéricos y concentrados en una opción de acción pre-configurada - un producto o resultado bueno para todos. Los resultados esperados con la consejería, por lo tanto, no pueden ser estáti$\cos y$ fijos, sino construidos conjuntamente con el individuo; son inéditos y variables, lo que permite la surjan soluciones innovadoras, originales y articuladas con el contexto social, cultural y con las condiciones afectivo-emocionales del individuo. Eso solo incrementa las posibilidades de manejar y e superar los obstáculos de la prevención y de la asistencia.

En esta visión de la relación de ayuda, dichos resultados se refieren también a la persona en su integralidad, es decir, son cambios relativos principalmente a la forma en que el individuo percibe y se posiciona con relación a sí mismo y a su realidad. Estos cambios se relacionan a una ampliación del campo perceptivo y de conocimiento sobre determinada situación/ problema y a un incremento del control y del dominio del individuo sobre esa misma condición, área o situación de vida - y sobre las oportunidades y recursos existentes.

Los principales resultados alcanzados por los individuos que participaron de los procesos de ayuda fueron descritos por Rogers (1981, 2001a) de la siguiente forma: (a) mayor apertura hacia la propia experiencia, es decir, más disponibilidad y capacidad de acceder, visualizar, aceptar y comprender los distintos elementos objetivos y subjetivos presentes en una situación determinada; (b) visión más realista de sí mismo y de su realidad; (c) menos rigidez y más flexibilidad para ver y manejar la realidad; (d) mayor apertura a cambios de actitudes y a transformaciones en su propia vida; (e) más auto-dirección, autonomía y responsabilidad por sí mismo; (f) visión y expectativas más realistas de la personas a su alrededor.

Esta visión de resultados esperados identificada en el abordaje de Rogers amplia de forma considerable el horizonte normativo de la consejería, haciendo con que la expectativa en relación a la atención sea más abarcadora, y al mismo tiempo, más dependiente de la singularidad y de la disponibilidad individual. Aún como parte de esta perspectiva, se encuentra el compromiso del profesional no apenas con un problema determinado, una política, o con superar un mal colectivo, aunque ese norte pueda participar en la atención llevada a cabo, pero introduce también, y principalmente, un compromiso con la felicidad humana, con lo que Rogers llama de "buena vida" (Rogers, 2001a).

En lo que respecta los objetivos y resultados, este estudio encontró algunas contradicciones, oposiciones y contrastes entre la racionalidad del Abordaje Centrado en la Persona y la racionalidad de la consejería en EST/Sida, tal como se delinea en los manuales nacionales e internacionales relevados.

Una primera oposición se relaciona al hecho de que la consejería propuesta por los manuales tiene su propia estructura operacional (el cómo hacer) dirigida a un camino más dirigido, cerrado, coordinado por metas de salud colectiva. La consejería en EST/Sida, especialmente a partir de 1997, pese a haber sido mencionado como una relación de ayuda centrada en el individuo, 
acaba por definir objetivos que no son totalmente acordes con esta concepción.

En todos los manuales nacionales e internacionales, aunque de distintas maneras ${ }^{2}$, los objetivos de la consejería en EST/Sida definen y mezclan metas y preocupaciones comprometidas con el plan individual, pero abiertas y enfocadas en la singularidad y el dinamismo de la persona en cuestión, y metas colectivas y sociales, conformadas por un ideal de bien estar social, con una visión global de la vida sana, muy a menudo distante de la realidad individual. Las dos metas tienen la intención de ser alcanzadas igualmente por la consejería y se dirigen de forma simultánea al individuo en cuestión, sugiriendo que el misma sea abordado tanto desde la perspectiva normalizadora y genérica sobre su comportamiento, como por una perspectiva que fortalece y reconoce su autonomía y su singularidad.

De esta forma, se proponen metas más personalizadas e indeterminadas, tales como: la reducción del nivel individual de estrés; la reflexión y la auto-comprensión personal que propicia la percepción de los propios riesgos y la revisión de su posicionamiento ante estos; el establecer condiciones favorables para que el individuo identifique y haga frente a situaciones adversas y pueda tomar decisiones; el empoderamiento y fortalecimiento de la autonomía del individuo; el apoyo y expresión emocional para poder enfrentar las dificultades y asuntos dificiles; y fomentar la auto-confianza individual. Estos objetivos, no obstante, conviven con otros más generales, colectivos y pre-configurados, tales como: la ruptura de la cadena de transmisión de las EST y el VIH; adopción de prácticas consideradas seguras o un cambio en el comporta-

\footnotetext{
Los manuales internacionales subrayan la importancia de la flexibilidad en los objetivos propuestos para cada sesión de consejería, apuntan a la equivocación de usar dicha sesión para colectar datos para el servicio o para suministrar información estandarizada. Advierten contra la tendencia de "encajar" a la personas en un mismo mensaje de prevención, desafían a los profesionales para que accedan al contexto y a la perspectiva afectivo-emocional y socio-cultural de los individuos (CDC, 2001; WHO, 1995b).
}

miento de riesgo; comunicación y tratamiento de las parejas; adherencia al tratamiento; y el fortalecimiento de informaciones actualizadas sobre las EST/HIV/Sida (MS, 1997; WHO, 1995a, 1995b).

Son esos los dos focos de acción que se traducen en compromisos distintos, además de configuran como dos racionalidades diversas en la construcción y conducción de la atención, la del apoyo individual y el compromiso con los procedimientos y demandas epidemiológico-sanitarias, es decir, con las políticas universales de prevención y ayuda a las EST/Sida. Como nos advierten otros autores que analizan la consejería en EST/Sida, pese a que exista un espacio abierto para el diálogo, existe cierta rigidez en este supuesto "diálogo", en la medida en que los proyectos personales se someten a la racionalidad de acciones públicas (Souza \& Czeresnia, 2007).

En aquellas situaciones en que el principal objetivo es la ejecución de una política, la persona, en sus distintas dimensiones, con sus necesidades, deseos y proyectos de vida, tiende a ser relegado a un segundo plano. Con esta pauta y agenda colectivas, y el discurso de carácter más universal y normativo, resulta mucho más difícil incorporar la propuesta de una acción enfocada en la evaluación particular de cada contexto y realidad individual. El énfasis en protocolos, conocimientos y procedimientos estandarizados con frecuencia va a contramano de la postura necesaria para acceder a experiencias, percepciones y poder entender al individuo en cada una de las situaciones. Promover e incentivar un determinado comportamiento y una acción pre-fijada apunta hacia una dirección distinta a la de ayudar al individuo a entenderse a sí mismo y tomar libremente sus decisiones personales.

\section{Papel y Postura del Profesional}

Según Rogers, uno de los elementos clave para poder construir una práctica de ayuda eficaz y resolutiva es la propia actitud y postura que asume el profesional en esta atención. La visión que tiene el profesional de su papel en esta relación determina como va a conducir su atención, sus prioridades, las técnicas utilizadas, su 
expectativa con relación al comportamiento del individuo al que quiere ayudar y el espacio que le concede en esa relación (Rogers, 1981).

Además, la construcción de una relación de ayuda en esta perspectiva se basa fundamentalmente en establecer condiciones interpersonales e intersubjetivas favorables a la auto-percepción, auto-exploración, auto-concientización y auto-determinación del sujeto en lo que respecta su realidad.

Un primer aspecto que amerita ser mencionado se refiere a la postura y actitud del profesional propuesta por Rogers es su disponibilidad para la auto-restricción, es decir, su abstención adrede de convertirse en el centro y en el elemento que sirve de norte a la acción, así como de utilizar el poder conferido por su propia formación y rol profesional, para avergonzar al otro en determinados comportamientos $\mathrm{u}$ opiniones (Rogers, 1981).

Siendo así, pese a que el profesional siempre tenga la intencionalidad y una expectativa propia - de como disminuir el riesgo de la infección, mejorar la calidad de vida o mostrar la relevancia y la servidumbre a ciertas estrategias preventivas $-\mathrm{y}$ aunque pueda, en algunas situaciones, exponer esa intencionalidad y expectativa al otro, la acción propuesta por Rogers no se basa en un actuar estratégico (uno actuando sobre el otro). Se acerca más bien a un actuar comunicativo (un actuar con el otro), que busca acceder y entender los motivos y razones del otro, para después articular perspectivas y posibilidades de derivación que tengan sentido dentro de la totalidad de la existencia del otro. ${ }^{3}$

3 En el actuar estratégico, según Habermas, el lenguaje aparece tan solo como medio de transmitir informaciones, de convencimiento y persuasión, el efecto de coordinación de la acción ocurre por medio de influencias recíprocas en que los actores persiguen objetivos. La interacción se basa en convicciones monológicas y no se logra establecer un vínculo de reciprocidades que caracteriza la posibilidad de un acuerdo y entendimiento mutuo. Por otra parte, en la acción comunicativa, el lenguaje aparece como generador de entendimiento, fuente de integración social. Se trata de conocer y entender las razones del otro, se intenta entender una situación particular (Boufleuer, 2001).
Esta perspectiva propuesta por Rogers trae a luz un nuevo movimiento y posicionamiento ante las acciones de prevención. Cualquier acción de prevención y cuidado discutida en la consejería necesita estar articulada y ser permeable a las circunstancias de vida, a los valores y necesidades consideradas importantes por ese otro.

En la misma dirección, Ayres (2004), al discutir las implicaciones de repensar y de reconstruir las prácticas de salud, para buscar desarrollar acciones que puedan ir más allá de la a "mera reproducibilidad técnica", sugiere que el profesional debe estar abierto a soluciones preventivas y acciones de cuidado más singulares y heterodoxas, que huyen del discurso pre-modelado, del tipo: "todos usen preservativo, siempre, en todas las relaciones sexuales". Debe poder usar la escucha precisa y la creatividad conjuntamente para ayudar al individuo construir, paulatinamente soluciones viables y que tengan sentido dentro de su realidad, aunque sea una solución inesperada y distinta de la habitual.

Otro aspecto relevante identificado en este abordaje teórica involucra no iniciar la relación de ayuda con una visión pre-definida del otro, lo que significa no encuadrar las personas atendidas en ninguna apreciación, clasificación o definición fija. Eso implica no dejar que el pasado o el presente de las mismas, que determinado diagnóstico, que análisis socio-conductuales anteriores de colectividades específicas, o que determinado contexto restrinjan la capacidad del profesional de oír y comprender la singularidad de cada sujeto y de los contextos individuales, o que lo impidan de tener acceso, a partir del dialogo intersubjetivo, a lo que el otro está realmente experimentando y aportando a la relación. De esta forma, propone que no se lleve los sentidos listos, definidos y construidos y que uno esté abierto a nuevas, improbables y hasta sorprendentes formas de entender y enfrentar las cosas.

Todo ello significa no encarcelar al otro en una categoría nosológica particular, o en un grupo social especifico, reduciéndolo, por ejemplo, o a un portador de una infección, a un miembro de una población vulnerable, o a un portador de un comportamiento de riesgo. Implica mirar al otro como un ser dinámico, en cons- 
tante transformación, un sujeto que se construye a cada instante en sus contactos con el mundo, una "carencia de lo mismo" (Ayres, 2001).

Rogers siempre manifestó ser en contra de cualquier tipo de diagnóstico bio-psico-social del individuo que lo enmarcara en alguna tipología genérica. No se debe, por lo tanto, transponer sin críticas al plan individual características comportamentales y socio-culturales de colectividades y grupos. El análisis del individuo es singular y transitorio, y cambia cada vez que nos encontramos con la misma persona. Eso exige una postura de apertura una vez más, no de clasificación (Rogers, 2001a).

"Dejar al otro convertirse y estar en constante cambio, no encarcelarlo en una apreciación, clasificación, o definición fija específica, que se cristaliza en algún aspecto de su presente o pasado" (Rogers, 2001a, p. 65).

Para no encuadrar al otro, y con la intención de permitir que el cliente se sienta seguro y libre para explorar mejor y para entender su experiencia presente, Rogers muestra cuán saludable es, incluso para el profesional, tener una posición que restrinja al máximo la necesidad humana inherente y natural de clasificar y pre-encasillar los hechos. En base a sus estudios sobre las relaciones humanas y los procesos de comunicación, considera que una de las principales barreras a la comprensión mutua y a la comunicación interpersonal es la tendencia humana de pre-organizar la realidad, siempre juzgando y valorando los hechos de forma positiva o negativa (lo que aporta un sentimiento de seguridad, control y dominio de la situación), sin permitirse ver cuidadosamente la realidad bajo otros aspectos y ópticas (Rogers, 2001a, 2001b).

La principal barrera a la comunicación interpersonal es nuestra tendencia muy natural de juzgar, evaluar, aprobar o desaprobar las afirmaciones de otra persona o de otro grupo, analizándolas a partir de nuestro propio marco de referencia, sin entender su sentido o significado para el otro. (Rogers, 2001a, p. 382)

Otra característica importante de la postura del profesional dentro de la concepción de Rogers de atención es la necesaria involucración, implicación, disponibilidad e interés del profe- sional en la persona del otro. Esto implica en que debe existir la disposición afectiva en la relación que facilita poder entablar esa relación interpersonal. Especialmente porque esta acción no es una tarea mecánica, sino más bien una vivencia y una experiencia racional y motivada afectivamente (Rogers, 2001b).

En la atención, el profesional hace uso no apenas de sus conocimientos especializados (de una racionalidad pre-orientada y dirigida), y ni se apoya en la ejecución rigorosa de una atribución estipulada o en preservar un imagen inviolable. Este debe estar abierto a utilizar todos los elementos presentes en su personalidad en la lectura de la situación, y dispuesto a descubrir y a construir conjuntamente con el otro, los sentidos y los significados posibles en la relación.

En ese sentido, el profesional no solamente aproxima su escucha del otro, sino que acerca igualmente la escucha de sí mismo frente al otro, dejándose "ser tocado" por ese otro, e identificando el impacto que cause en él la presencia del otro. Como nos afirma Ayres (2004), cuando discute la posibilidad de más autenticidad y efectividad en el encuentro terapéutico, este tipo de disposición provoca una inflexión en el formato tradicional formato de la atención, otorgándole prioridad a la escucha precisa y a la dimensión dialógica del encuentro: el diálogo del profesional con la persona que busca ayuda, de esta persona consigo misma y del profesional consigo mismo.

Adicionalmente a esta apertura, disponibilidad y receptividad hacia el otro, Rogers destaca la importancia de reconocer al otro en su integralidad, en sus diferencias, y en sus ambigüedades, es parte de reconocer los distintos tipos de vivencias, contenidos y valores individuales. Esto implica aceptar como legítimas todas las reacciones, sentimientos y pensamientos del sujeto que busca ayuda. De esa manera, todos los tipos de vivencias y contenidos (agradables o desagradables, constructivos y destructivos, interesantes o no, claros e confusos) deben tener su espacio en la relación. La persona que pasa por la consejería debe tener su existencia aceptada por entero, tal como verdaderamente es, o como se presenta en ese momento, con sus ambigüedades, contradicciones e idiosincrasias (Rogers, 2001a). 
Así, no se trata de adecuar, alterar, negar, distorsionar o minimizar la vivencia y el sentimiento que trae el otro, dándoles un sentido, una explicación e interpretación previa y lista, de tal forma que se hagan más palatables y menos incómodos (como por ej: "tú no puedes estar sintiéndote tan abandonado así, pese a que no hables con tu esposa, porque tú mismo has dicho que aún tienes amigos", o "tu no necesitas tanto temer hacer ese examen, ya que es siempre mejor conocer tu situación serológica para poder cuidarte y minimizar las consecuencias, cuanto antes", o "porque te sientes tan mal y tan molesto al usar condones? Estoy convencido de que puedes acostumbrarte a ellos, tan solo tienes que intentarlo"). Se deja que la propia exploración de la experiencia revele su significado. Así, se permite que el odio sea odio, la vergüenza sea vergüenza y el sentimiento de impotencia y destrucción sean lo que son, y en esa experiencia se busca los propios motivos, consecuencias, posibilidades de hacer frente al problema y a las interpretaciones que se dan a éste.

Esta condición implica que el terapeuta esté realmente listo a aceptar al cliente, independientemente de lo que esté sintiendo el cliente, y viviendo en el momento: cólera, confusión, miedo, disgusto, orgullo, desánimo, irritación, ironía, falta de interés, coraje, admiración. Significa que el terapeuta se preocupa con el individuo de forma no posesiva y auto-centrada, que lo aprecia más en $s u$ totalidad que de una forma condicional, que no se contenta con aceptar simplemente a su cliente cuando éste sigue determinados caminos y lo desaprueba cuando sigue otros. (Rogers, 2001a, p. 72)

Este ambiente de respeto, reconocimiento y apertura a las distintas facetas de la existencia humana del otro disminuye ese juicio moralista y crea un ambiente más protegido, libre y confiable, que facilita la auto-confrontación, la posibilidad de autocrítica y revisión de actitudes. Esta actitud de aceptación no es mecánica o enseñada, es más bien una disposición que puede ser experimentada y aprendida y que no ocurre en todos los momentos, pero, de acuerdo a Rogers, es una disposición extremadamente facilitadora para que cada persona se sienta segura y motiva- da a explorar mejor la naturaleza y los contornos de su problema.

Para lograr hacer eso, el profesional debe aceptar del otro cualquier tipo de experiencia vivida (rabia, inseguridad, desprecio, descrédito, ansiedad), sin que se sienta amenazado, frustrado, cobrado e irritado como profesional. Necesita una cierta dosis de humildad y percepción de límites (dejar de lado la postura omnipotente y controladora), un cierto fortalecimiento, aceptación y conciencia de la propia identidad personal, distinguiéndola del individuo ante él, para no imaginar que la elección del otro depende estrictamente de sus propias reacciones, deseos y características personales.

Podré ser suficientemente fuerte como persona, para ser independiente del otro y dejar que el otro sea independiente de mi? Seré capaz de respetar valientemente mis propios pensamientos y sentimientos, así como los de la otra persona? Seré lo suficientemente fuerte en $m i$ independencia para no deprimirme con su depresión, asustado con su miedo o involucrado en su dependencia? Será mi fuero interno lo suficientemente fuerte para sentir que yo no soy ni destruido por su cólera y miedo, ni absorbido por su necesidad de dependencia y de respuestas rápidas, ni esclavizado por su afecto, pero que existo independientemente de la reacciones del otro? (Rogers, 2001a, p. 61)

Una última condición, considerada por Rogers como absolutamente clave e indispensable para una relación de ayuda es la postura de escucha y comprensión empática. La comprensión empática es, en primer lugar, una actitud de aproximación deliberada del marco de referencia interno del otro, es decir, un acercamiento de la síntesis dinámica y personal que hace el individuo del horizonte normativo que le sirve de norte (valores, tradiciones, creencias, reglas, suposiciones, reglas y normas sociales), de tal forma a entender mejor las distintas facetas que existen en el campo perceptual del individuo (significados y valores atribuidos), tal como éste los comunica (Rogers, 2001a, 2001b, 2005b).

En un segundo momento, la actitud de comprensión empática significa devolver y comunicar con la persona que busca ayuda, todo lo que 
el profesional se ha percibido y entendido en su actitud de examinar y escrutar los significados personales dados a situaciones específicas de la vida, para poder ampliar y aclararla percepción del cliente, a respeto de sí mismo.

En un tercer momento, la comprensión empática implica verificar y chequear y evaluar con cada persona la precisión y la exactitud de la lectura que ha hecho el profesional de las vivencias del mismo, siempre reformulando y cerciorándose de la validez y legitimidad de dichas interpretaciones a partir de las respuestas (confirmaciones o discordancias) obtenidas con él.

Esa actitud de aproximación deliberada al marco de referencia del otro no es intuitiva e inmediata, sino mediada por la comunicación, es decir, por la exposición verbal, explicación y juicio que hace el individuo mismo de sus vivencias. Así, pese a que la experiencia subjetiva sea una actividad individual, la comprensión de la misma es una actividad de comunicación. Los sentidos y significados suministrados son construidos y expresados inevitable y constantemente por medio del lenguaje en acto. Hay por lo tanto siempre una intersubjetividad que tiene que suponerse, puesto que existe una comunicación y una reconstrucción de sentidos.

Rogers ponderó que solo era posible alcanzar el marco de referencia del otro, primero, porque éste puede ser comunicado lingüísticamente $\mathrm{y}$, segundo, porque existe un substrato de experiencias y vivencias comunes - que para Rogers están relacionadas principalmente a la experiencia del humano, pues este substrato no se concentra en cuanto es construido por la cultura (Rogers, 2001b).

Es pues por medio del lenguaje que llegamos al otro. Para ello el profesional debe indagar al otro sobre los significados y pesos que se dan a los hechos, contextos y a cada situación y escena relatada. La esencia de este tipo de relación de ayuda yace en la auto-comprensión mutua de las razones y significados, y no en el convencimiento; o sea, se encuentra en reconocer las motivaciones, significados y consecuencias de cada una de las cuestiones y situaciones expuestas por el cliente.

Los manuales internacionales de consejería en EST/Sida investigados, y especialmente el
"Source Book for HIV/AIDS Counseling Training" (WHO, 1995b), muestran una preocupación mayor que los nacionales en cualificar las posturas de los profesionales y el manejo de la consejería, presentan de forma mucho más ahondada valores, técnicas y posturas que deben desarrollarse en esta acción, e incluso discuten características, contornos y especificidades de la comunicación interpersonal como siendo elementos fundamentales para esa acción: postura de buscar la interpretación personal de los individuos sobre los problemas planteados; alentar y facilitar la expresión de sentimientos; reconocer y distinguir las varias emociones y percepciones experimentadas; ayudar a los individuos a identificar y mejor delimitar sus demandas; ayudarlo a explorar las creencias y significados sobre los asuntos en discusión; aceptar y reconocer como válidas las diferencias en las elecciones, soluciones y valores individuales; mostrar las relaciones entre las actitudes y posturas del individuo y sus consecuencias en el entorno y para sí mismo; confrontar al individuo con las inconsistencias en su historia, comportamiento o discurso; darle la oportunidad de redimensionar sus dificultades, sentimientos y conocimientos; entre otras (WHO, 1995a, 1995b).

Los manuales nacionales de consejería, especialmente el documento que fue publicado en 1997 y re-editado un sin número de veces (MS, 1997), no se enfocan en el manejo de la consejería, no discuten las condiciones adecuadas para su puesta en práctica, ni discurren sobre posturas, actitudes de los profesionales y formas de conducción. En dichos manuales no existe ningún rubro que discurra y analice habilidades, posturas y técnicas de manejo de esta relación interpersonal. Por ese motivo, no proveen los elementos necesarios para calificar y ahondar más en ese abordaje interpersonal y el manejo técnico de esta actividad. Se centran en sugerir procedimientos que se deben llevar a cabo, pero no ahondan en el significado y la puesta en operación de los mismos - ayudar al cliente a evaluar y percibir sus riesgos; facilitar la expresión de sentimientos; contribuir a la elaboración de un plan factible para la reducción de riesgos, etc. Se concentran además en sugerir contenidos que se deben abordar - informaciones sobre EST, 
informaciones sobre la prueba y la ventana inmunológica, etc. Eso lleva a una actuación más mecánica, estandarizada y formateada, o a una actuación más intuitiva de esta práctica.

En encuesta realizada en 1995 por la Coordinación Nacional de EST/Sida, sobre la evaluación de las acciones de la consejería realizadas en distintos servicios de EST/Sida (Filgueiras \& Deslandes, 1999), entre los principales problemas encontrados estaban: postura de distanciamiento en lo que respecta las expectativas y vivencias del usuario; inseguridad en lidiar con situaciones inesperadas; sensación de no estar preparado para el manejo de aspectos afectivo-emocionales; postura de "preguntador", siendo que las respuestas del usuario no servían como elementos para el diálogo; repetición de preceptos normativos para la prevención; priorización de un guión desperdiciando diversas oportunidades para la reflexión sobre riesgos y actitudes individuales.

No obstante, como es posible personalizar y contextualizar las informaciones ofrecidas y ayudar a pensar en estrategias y elecciones que dialoguen con la vivencia que tiene el individuo de su contexto o que se ajusten y se articulen con su proyecto de vida, si hay poco espacio para acceder a y entender la dinámica individual?

\section{Consideraciones Finales}

\section{Caminos para Superar los Callejones u Obstáculos Identificados}

Un primer impase identificado en este estudio se refiere, como ya lo vimos, al hecho de que la consejería propuesta por los manuales de consejería que existen en el Brasil tiene su estructura conceptual, valorativa e ideológica inspirada en el Abordaje Centrado en la Persona (la naturaleza de ese trabajo, en nombre de qué hacer, para qué y para quine hacerlo); pero su estructura operacional (cómo hacer) va rumbo a otra dirección (más directiva, cerrada y coordinada por metas de salud colectiva).

La técnica propuesta para la consejería en EST/Sida acaba por tener una configuración confusa, haciendo con que la práctica efectiva de la consejería pueda oscilar por veces en una direc- ción, por veces en otra, dependiendo del perfil, del interés y de la disponibilidad del profesional. El discurso del colectivo, pautado en el saber epidemiológico, preventivo y médico-sanitario (con su urgencia de controlar la epidemia y luchar lo antes posible contra todas las posibilidades de riesgo e infección), en el cual los propósitos son abarcadores, genéricos e uniformes y los problemas y soluciones son más homogéneos y estructurados, siempre necesitará ese cambio de perspectiva al transponerse al plan individual.

Es menester subrayar que estos dos modos de aproximarse a la realidad (foco en metas colectivas o en metas individuales), así como las acciones que resultan de éstos, son ambos pertinentes e indispensables para el campo de la salud y para el control de la epidemia. Sin embargo, tal vez no todos necesiten alcanzarse y ser resueltos en una misma práctica específica. En la consejería se puede y se debe hacer frente a las demandas y cuestionamientos más macro y generales, pero siempre a nivel micro y particular. Este particular, no es único, unido y uniforme, sino con múltiples facetas, diversificado, dinámico e inconstante. Los protocolos de prevención y asistencia, aunque sean útiles y relevantes, no son suficientes para acceder a la gran diversidad de esa realidad. Con frecuencia es necesario que se readecúen y se adapten a las condiciones y circunstancias individuales, así como que se acuerden intersubjetivamente.

Con el fin de tratar de buscar algunas posibilidades para superar los posibles obstáculos y fragilidades identificadas en la práctica del la consejería en EST/Sida en base a este estudio, y para hacer un uso consistente de algunos subsidios identificados en el Abordaje Centrado en la Persona, es imperioso notar que no todo debe resolverse en la consejería. La consejería jamás debe ser considerada como una acción aislada, sino que debe siempre inserirse en una estrategia más amplia de prevención y asistencia, en donde se suman otros tipos de intervención (individual y colectiva) que lleguen a los individuos y a los grupos sociales de formas diferentes. Las estrategias deben ser tanto globales como particulares y las respuestas y soluciones deben ser tanto comunitarias como individuales. 
Para garantizar una oferta inteligente y coherente de consejería en los diferentes espacios en donde se utiliza, es indispensable que todos los profesionales que hacen uso de esta tecnología de ayuda entiendan la naturaleza, las especificidades y la finalidad de la misma, para poder saber cuándo y cómo utilizarla bien, y por lo tanto, saber diferenciarla de otras acciones del servicio (el llenado de fichas y protocolos, entrega de resultados, actividades educacionales colectivas, acogimiento etc.).

Es necesario además recordar que la consejería es una práctica que, desde su origen, ha trabajado en el ámbito de la realidad y del universo individual y no en el ámbito del universo de lo colectivo. Involucra siempre la posibilidad de que el individuo vislumbre, evalúe, se exprese y se posicione sobre su propia realidad. Es por ello, por su misma naturaleza y características, una estrategia personalizada y particularizada, que busca abordar cualquier situación específica siempre en relaciónala totalidad de la existencia del individuo, y no una ayuda genérica, ya lista y pre-configurada.

Los manuales de la Organización Mundial de la Salud (WHO, 1995a) dejan claro que la principal característica de la consejería es la de ser una acción dirigida especialmente al manejo de los aspectos socio-culturales y afectivo-emocionales del diagnóstico, prevención y tratamiento de las EST/HIV/Sida (WHO, 1995a). Hay, inherente a esa práctica, la gran necesidad de habilidades interpersonales, habilidades de escucha y comunicación, así como habilidades para acceder a y manejar emociones, sensaciones y significados particulares, y no apenas el dominio de informaciones técnico-científicas. Así, se debe aprovechar la riqueza y las posibilidades que ese tipo de espacio restringido al campo interpersonal ofrece.

Dentro de esa perspectiva, este estudio nos insta a calificar el ejercicio de la consejería, para que esa actividad no sea una acción burocratizada, de rutina, estandarizada, "igual para todos". Se debe reforzar la propuesta de que se incorpore a las estrategias de respuesta a la epidemia del Sida como una tecnología para ayudar a las personas, que favorezca el aumento de su percepción sobre la cuestión del riesgo, infección o padecimiento, y las potencialice para realizar cambios convenientes y relevantes en su propia vida.

Para eso sería necesario sin embargo que la consejería dejase de ser un espacio obligatorio para convertirse en una actividad voluntaria, ofrecida de forma inteligente, comprensible, accesible y cuidadosa, a todos los individuos que necesitasen y se interesasen por ese tipo de apoyo.

En esa misma dirección, la consejería tendría que dejar bajo su cargo la ejecución de procedimientos, la comunicación de contenidos específicos o la colecta de informaciones sobre perfiles de riesgo.

Finalmente, pero no menos relevante, cabe hacer hincapié en el hecho que tal cambio exigiría también una atención especial en relación a los profesionales responsables por su ejecución, con una formación más consistente, con el desarrollo de actitudes y posturas volcadas a la comunicación y a la escucha (no moralista) del otro, así como un espacio de supervisión y seguimiento necesarios para el bien estar del profesional y para la calidad de su trabajo.

\section{Referencias}

Ayres, J. R. C. M. (2001). Sujeito, intersubjetividade e práticas de saúde. Ciência e Saúde Coletiva, 6(1), 63-72.

Ayres, J. R. C. M. (2004). O cuidado, os modos de ser (do) humano e as práticas de saúde. Saúde e Sociedade, 13(3), 16-29.

Boufleuer, J. P. (2001). Pedagogia da Ación Comunicativa. Ijuí, RS: Editora da Universidade Regional do Noroeste do Estado do Rio Grande do Sul.

Castrucci, B. C., Kamb, M. L., \& Hunt, K. (2002). Assessing the Center for Disease Control and Prevention's 1994 HIV Counseling, Testing, and Referral: Standards and Guidelines (How closely does practice conform to existing recommendations?). Sexually Transmitted Diseases, 29(7), 417-421.

Centers for Disease Control and Prevention. (1993). Technical Guidance on HIV Counseling, Testing e Referral: Standards and Guidelines. Atlanta, GA: Author. 
Centers for Disease Control and Prevention. (1994). Technical Guidance on HIV Counseling, Testing e Referral: Standards and Guidelines. Atlanta, GA: Author.

Centers for Disease Control and Prevention. (2001). Revised Guidelines for HIV Counseling, Testing and Referral and Revised Recommendations for HIV Screening of Pregnant Women. Morbidity and Mortality Weekly Report, 50.

Corey, G. (1997). Counseling and psychotherapy: Theory and practice. Washington, DC: Brooks/ Cole

Dolcini, M. M., Canin, L., Gandelman, A., \& Skolnik, H. (2004). Theoretical domains: A heuristic for teaching behavioral theory in HIV/STD prevention courses. Health Promotion Practice, 5(4), 404-417.

Filgueiras, S. L., \& Deslandes, S. F. (1999). Avaliación das ações de consejería: análise de uma perspectiva de prevención centrada na pessoa. $\mathrm{Ca}$ dernos de Saúde Pública, 15(Supl. 2), 121-131.

Gadamer, H. G. (2004). Verdade e método I: Traços fundamentais de uma hermenêutica filosófica (6. ed.). Petrópolis, RJ: Vozes.

Grinstead, O. A. (1997). HIV Counseling for Behavior Change. Aids Education and Prevention, 9(2), 125-132.

Holtgrave, D. R., Reiser, W. J., \& Franceisco, W. D. (1997). The evaluation of HIV Counseling and Testing Services: Making the most of limited resources. AIDS Education and Prevention, $9(\mathrm{Su}-$ ppl. B), 105-118.

Joint United Nations Programme on HIV/AIDS, \& World Health Organization. (2000). Opening up the HIV/Aids Epidemic: Guidance on encouraging beneficial disclosure, ethical partner counseling and appropriate use of HIV case Reporting (UNAIDS/0042E). Geneva, Switzerland: Joint United Nations Programme on HIV/ AIDS.

Joint United Nations Programme on HIV/AIDS, \& World Health Organization. (2001). The impact of Voluntary Counseling and Testing: A global review of the benefits and challenges (UNAIDS/01.32E). Geneva, Switzerland: Author.

Joint United Nations Programme on HIV/AIDS. (2000a). Tools for evaluating HIV voluntary counseling and testing. Best Practice Collection. Geneva, Switzerland: Author.
Joint United Nations Programme on HIV/AIDS. (2000b). Voluntary Counseling and Testing (VCT) Technical Update. Geneva, Switzerland: Author.

Kirschenbaum, H., \& Henderson, V. L. (Eds.). (1989). The Carl Rogers Reader - Selections from the Lifetime Work of America's Preeminent Psychologist. New York: Houghton Mifflin.

Lewis, E. C. (1970). The Psychology of Counseling. New York: Holt, Rinehart and Winston.

Ministerio de la Salud. (1988). Manual de Condutas Clínicas. Brasília, DF: Autor.

Ministerio de la Salud. (1989). Manual de Consejería/SIDA. Brasília, DF: Autor.

Ministerio de la Salud. (1993). Normas de organización e funcionamento dos Centros de Orientación e Apoio Sorológico. Brasília, DF: Autor.

Ministerio de la Salud. (1994). Relatório do I Encontro de Avaliación de Centros de Apoio Sorológico. Campinas, SP: Autor.

Ministerio de la Salud. (1996a). Relatório do II Encontro de Avaliación de Centros de Apoio Sorológico. Brasília, DF: Autor.

Ministerio de la Salud. (1996b). Relatório do Seminário de Consejería. Brasília, DF: Autor.

Ministerio de la Salud. (1997). Consejería em EST, HIV e Sida-diretrizes e procedimentos básicos. Brasília, DF: Autor.

Ministerio de la Salud. (1998). Manual de Treinamento de Consejería em EST, HIV e Sida. Brasília, DF: Autor.

Ministerio de la Salud. (1999). Consejería: um desafio para a prática integral em saúde-avaliación das ações. Brasília, DF: Autor.

Ministerio de la Salud. (2000). Diretrizes dos Centros de Testagem e Consejería (CTA). Brasília, DF: Autor.

Ministerio de la Salud. (2004). Consejería em EST/ HIV/Sida para a Atención Básica. Brasília, DF: Autor.

Ministerio de la Salud. (2005). Oficina de Consejería em EST/HIV/Sida para a Atención Básica. Brasília, DF: Autor.

Myers, T., Worthington, C., Haubrich, D. J., Ryder, K., \& Calzavara, L. (2003). HIV testing and counseling: Test providers' experiences of test practices. AIDS Education and Prevention, 15(4), 309-319. 
Pattersson, C. H. (1973). Theories of Counseling and Psychotherapy. New York: Harper and Row.

Pattersson, E. L., \& Eisenberg, S. (2003). O Processo de Consejería. São Paulo, SP: Martins Fontes.

Rogers, C. R. (1981). Psicoterapia centrada en el cliente. Buenos Aires, Argentina: Paidós.

Rogers, C. R. (2001a). Tornar-se Pessoa. São Paulo, SP: Martins Fontes.

Rogers, C. R. (2001b). Sobre o Poder Pessoal. São Paulo, SP: Martins Fontes.

Rogers, C. R. (2005a). Psicoterapia e consulta psicológica. São Paulo, SP: Martins Fontes.

Rogers, C. R. (2005b). A pessoa como centro. São Paulo, SP: Editora Pedagógica e Universitária.

Santos, O. B. (1982). Aconselhamento psicológico e Psicoterapia. Auto-Afirmação - Um determinante básico. São Paulo, SP: Livraria Pioneira.

Scheeffer, R. (1976). Teorias de Consejería. São Paulo, SP: Atlas.

Sikkema, K. J., \& Bissett, R. T. (1997). Concepts, Goals and Techniques of Counseling: Review and Implications for HIV Counseling and Testing. AIDS Educations and Prevention, 9(Suppl. B), 14-26.

Souza, V., \& Czeresnia, D. (2007, set./dez.). Counseling discourse in anti-HIV testing services. Interface - Comunicação, Saúde, Educação, 11(23), 531-548.
Tyler, L. E. (1969). The Work of Counselor. Century Psychology Series. New York: Appleton Century Crofts.

Whiteley, J. M. (1999). The paradigms of Counseling Psychology. The Counseling Psychologist, 27(1), 14-31.

World Health Organization. (1990). Guidelines for Counseling about HIV Infection and Disease. AIDS Series 8. Geneva, Switzerland: Author.

World Health Organization. (1995a). Counseling for HIV/AIDS: A key to caring (WHO/GPA/TCA/ HCS/95.15). Geneva, Switzerland: Author.

World Health Organization. (1995b). Source Book for HIV/AIDS Counseling Training: Global Programme on AIDS (WHO/GPA/TCO/HCS/94.9). Geneva, Switzerland: Author.

World Health Organization. (2002). Increasing access to HIV Testing and Counseling: Report of a WHO Consultation, 19-21 November 2002, Geneva. Switzerland. Geneva, Switzerland: Author.
Recebido: $23 / 07 / 2012$

$1^{a}$ revisão: $27 / 11 / 2012$

$2^{a}$ revisão: 03/04/2013

Aceite final: 03/04/2013 